\title{
Metabolomics analysis reveals the metabolic and functional roles of flavonoids in light-sensitive tea leaves
}

Qunfeng Zhang ${ }^{1,2}$, Meiya Liu ${ }^{1,2}$ and Jianyun Ruan ${ }^{1,2^{*}}$

\begin{abstract}
Background: As the predominant secondary metabolic pathway in tea plants, flavonoid biosynthesis increases with increasing temperature and illumination. However, the concentration of most flavonoids decreases greatly in lightsensitive tea leaves when they are exposed to light, which further improves tea quality. To reveal the metabolism and potential functions of flavonoids in tea leaves, a natural light-sensitive tea mutant (Huangjinya) cultivated under different light conditions was subjected to metabolomics analysis.

Results: The results showed that chlorotic tea leaves accumulated large amounts of flavonoids with ortho-dihydroxylated B-rings (e.g., catechin gallate, quercetin and its glycosides etc.), whereas total flavonoids (e.g., myricetrin glycoside, epigallocatechin gallate etc.) were considerably reduced, suggesting that the flavonoid components generated from different metabolic branches played different roles in tea leaves. Furthermore, the intracellular localization of flavonoids and the expression pattern of genes involved in secondary metabolic pathways indicate a potential photoprotective function of dihydroxylated flavonoids in light-sensitive tea leaves.

Conclusions: Our results suggest that reactive oxygen species (ROS) scavenging and the antioxidation effects of flavonoids help chlorotic tea plants survive under high light stress, providing new evidence to clarify the functional roles of flavonoids, which accumulate to high levels in tea plants. Moreover, flavonoids with ortho-dihydroxylated B-rings played a greater role in photo-protection to improve the acclimatization of tea plants.
\end{abstract}

Keywords: Camellia sinensis, Flavonoids, Light sensitive, Metabolism, Photo-protection

\section{Background}

Flavonoids, the main secondary metabolites in plants, are the most important quality-related compounds, and they comprise $20-40 \%$ of the dry matter in young shoots of tea plant [1]. These flavonoid compounds also contribute to the color, taste and aroma of brewed tea. The contents of catechins (flavan-3-ol), flavonols, flavonol glycosides, anthocyanin and leucoanthocyanidin account for the majority of flavonoids in tea plant. The basic molecular structure / carbon skeleton of flavonoids is C6-C3-C6, and they are classified as mono-, di- and trihydroxylated based on their hydroxylated B-rings. The

\footnotetext{
* Correspondence: jruan@tricaas.com

${ }^{1}$ Tea Research Institute, Chinese Academy of Agricultural Sciences, Hangzhou 310058, China

${ }^{2}$ Key Laboratory for Plant Biology and Resource Application of Tea, the Ministry of Agriculture, South Meiling Road 9, Hangzhou, Zhejiang 310008, China
}

(c) The Author(s). 2017 Open Access This article is distributed under the terms of the Creative Commons Attribution 4.0 International License (http://creativecommons.org/licenses/by/4.0/), which permits unrestricted use, distribution, and reproduction in any medium, provided you give appropriate credit to the original author(s) and the source, provide a link to the Creative Commons license, and indicate if changes were made. The Creative Commons Public Domain Dedication waiver (http://creativecommons.org/publicdomain/zero/1.0/) applies to the data made available in this article, unless otherwise stated. sponse to environmental cues [2]. Numerous studies have shown that the biosynthesis of flavonoids (expression of structural genes, activity of some important enzymes and concentrations of flavonoids) increases under high light intensity $[2,3]$. However, sub-groups of flavonoids in tea plants can be differentially affected [4, 5]. The fundamental role of flavonoids to cope with light stress may rely on their many potential functions [6].

The flavonoids metabolic pathway has been widely accepted to be involved in the regulation mechanisms of plants to various stressful conditions [7]. Flavonoids are the main regulators of plant growth and defense, and they are induced and biosynthesized as the result of a long-term natural selection and acclimatization process [8-12]. The main physiological functions of flavonoids in tea plant are scavenging reactive oxygen species and increasing tolerance to adapt to environmental change, 
e.g., as antioxidants in photoprotection. The antioxidant activity of flavonoids is attributed to their reaction with free radicals as a hydrogen donor. However, flavonoids with different molecular structures have great differences in their antioxidant activity, which is highly correlated with the substituent positions and the amount of hydroxyl groups on the B-rings. The more hydroxylation of the flavonol, the more hydrogen atoms can be provided for binding free radicals, and the more antioxidant activity; e.g., the antioxidant activity of myricetin is higher than that of kaempferol. Moreover, the tautomeric interconversions of ortho-dihydroxylated B-ring flavonoids make them more efficient at dissipating excess energy [13] and scavenging reactive oxygen species $[6,14,15]$.

Camellia sinensis (L.) O. Kuntze cv. Huangjinya, a natural light-sensitive tea mutant, shows high levels of free amino acids and a low content of polyphenols, which improves the quality of brewed green tea and imparts a higher economic value to the chlorotic varieties than to the non-chlorotic varieties [16-19]. The young leaves of Huangjinya display a yellow color and show normal growth despite being chlorotic under high light conditions [20]. Moreover, Huangjinya shows a totally different metabolic response to light compared to the normal tea species. For example, as the main type of secondary metabolism in tea plant, the biosynthesis of flavonoids increases with increasing temperature and illumination [5]. However, the concentration of most flavonoids decreases greatly in Huangjinya leaves when they are exposed to light. The characteristics of specific genes and chemicals such as total polyphenols, total amino acids, and pigments biosynthesis in Huangjinya has been reported in the previous studies [17, 20]. Although less attention has been paid to the metabolic regulation of flavonoids in this mutant, such an analysis may help to clarify the mechanisms by which flavonoids highly accumulate in tea plants.

Light is one of the most important environmental factors, providing energy and external stimuli for growth and development in plants [21]. Changes in light intensity alter a complex set of molecular events within the chloroplast [5, 22]. However, light energy beyond the acceptable range of the reaction center causes light damage, leading to photoinhibition and decreasing the photochemical and carbon assimilation rates [23, 24]. Thus, photoprotection, either by scavenging harmful reactive intermediates or dissipating excess energy to protect cells from death under light stress, is essential for photosynthesis [25]. Chloroplast-located flavonoids [10, 26], phenylpropanoids [27] and anthocyanins [28] are effective sunlight attenuators which play vital roles in protecting chloroplasts when facing with a superabundant radiant energy. The key antioxidant function of flavonoids to excess ultraviolet radiation b (UV-B)/sunlight irradiance have been well documented [29-31]. These studies are further confirmed by the fact that flavonoids with an ortho-dihydroxy structure in the B-ring are preferentially accumulated, compared to flavonoids having a mono-hydroxy substitution [32,33]. The carotenoid or xanthophyll cycle also participates in protecting plants from high light intensity by thermal dissipation [34]. Moreover, organisms show chlorosis when the carotenoid biosynthesis pathway is disrupted [25] or is inhibited by norflurazon [35].

Herein, the hypothesis of the present work was that the metabolism of flavonoids, especially the 'different branch', may be regulated by genetic and environmental stimuli in light-sensitive tea leaves and that it is highly correlated with the potential function of flavonoids in tea leaves. An experiment was therefore designed in which samples of tea leaves from a light-sensitive mutant cultivated under two different light conditions were compared using metabolomics analysis.

\section{Methods \\ Plant material and shading treatment}

Rooted-cuttings of the natural mutant Camellia sinensis (L.) cv. Huangjinya, which was officially released as a clone in Zhejiang province in 2008 were obtained free of charge from the owner of the mutant Yuyao Deshi Tea Plantation (located in Yuyao county, Zhejiang province). Each of four seedlings were planted in pots $(\sim 10 \mathrm{~L}$ capacity) filled with commercial growth medium consisting of perlite, vermiculite, and peat at the Tea Research Institute, Chinese Academy of Agricultural Sciences (TRI, CAAS, N $30^{\circ} 10^{\prime}, \mathrm{E} 120^{\circ} 5^{\prime}$ ) in May of 2013. The pots were placed in open with full sunlight and watered regularly. In March 2014, 60 pots of tea plants with uniform young shoots were selected for the experiment: Half of the pots were treated with high-density polyethylene tape two-pin net (60\% sun-shading), and the remaining 30 pots were exposed to full sunlight for ten days. Samples (young shoots with bud and two expanding leaves) were randomly selected, frozen with liquid nitrogen and then stored in a $-70{ }^{\circ} \mathrm{C}$ ultra-freezer. The sampling was repeated six times each from shaded and unshaded plants.

\section{Ultra-performance liquid chromatography quadrupole time of flight mass spectrometry (UPLC-Q-TOF/MS) based metabolomics analysis}

All 12 obtained samples (six biological repetitions for the shaded and unshaded groups) were used for metabolomics analysis. The metabolites were extracted from young shoots using a mixture of $75 \%$ methanol and $1 \%$ formic acid as described by Zhang et al. [5]. A $2 \mu \mathrm{L}$ sample was injected into a UPLC-Q-TOF/MS (Waters, 
UPLC/Xevo G2-S Q-TOF) and separated with an HSS T3 column as described by Zhang et al. [5]. TransOmics software (Version 1.0; Waters) was applied for data preprocessing. Metabolite peaks were assigned to the accurate mass measurements using online metabolite databases as described in Zhang et al. [5], and the retention times were compared with those in the published literature [5]. A matrix was exported for further statistical analyses. The unit variance was scaled for further statistical analysis using SIMCA-P (version 13.0, Umetrics, Umea, Sweden). Unsupervised principal component analysis (PCA) and supervised projection to latent structure discriminant analysis (PLS-DA) were carried out to dissect the overall variance of metabolites and the composition differences of the samples, respectively. The combination of $\mathrm{p}(\mathrm{corr})$ and variable importance in the projection (VIP) values from the PLS-DA were used as a coefficient for metabolite selection (VIP $>$ 1.0 and $\mid \mathrm{p}($ corr $) \mid>0.5)$. Student's $t$-test $(P<0.05)$ and one-way analysis of variance (ANOVA) using the SPSS (version 15.0, SPSS Inc., Chicago, IL) were performed for statistical analysis.

\section{Quantitative real-time PCR analysis}

Total RNA was isolated using a plant RNA extraction kit (Tiangen, China). PrimeScriptTM RT reagent kit (TaKaRa) was applied to synthesize cDNA. Quantitative real-time PCR (qRT-PCR) was performed on the Applied Biosystems 7300 machine (Carlsbad, CA, USA). The primer pairs used for qRT-PCR are shown in Additional file 1: Table S1, and GAPDH was used as the reference gene. For each target gene, triplicate reactions were performed. Relative transcript levels were calculated against that of the internal control GAPDH using the formula $2^{-\Delta \Delta \mathrm{Ct}}$. All data are shown as the mean $\pm \operatorname{SD}(n=3)$.
Quantitative determination of chlorophylls, carotenoids, and catechins

To determine the contents of chlorophyll and carotenoids, leaf discs with an area of $86.59 \mathrm{~mm}^{2}$ were removed using a perforated metallic cylinder. Tea infusions were analyzed on a reverse phase high-performance liquid chromatographic (HPLC) system (Waters 2695) coupled to a diode array detector (Waters 2998) as described in [5]. Catechins were also quantified by HPLC, and the separations were performed using a C18 reverse-phase column $(250 \times 4.6 \mathrm{~mm}$ i.d., Phenomenex, Torrance, CA, USA) as described by Wu et al. [36].

\section{Tissue localization of phenolic compounds}

Samples prepared by standard freehand sectioning and stained with $1 \%(w / v)$ vanillin- $\mathrm{HCl}$ reagent were used to study the localization of phenolic compounds. Photos of the sample sections were taken by a microscope (XQT2, COIC) before and after staining [37].

The localization of flavonoids was determined by staining the sections with NaturstoVreagenz $A$ and observing using confocal laser scanning microscopy (CLSM, Zeiss LSM 710 NLO) by the method described in [38].

\section{Results}

Phenotype and content of chlorophyll and carotenoids in Huangjinya leaves

As shown in Fig. 1, the leaves were chlorotic when the tea plants were exposed to full sun light condition, whereas the leaves of shaded tea plants turned green. Accordingly, the contents of chlorophyll in these two types of tea plant leaves were distinct (Table 1). Compared to the non-chlorotic tea plants, chlorotic leaves had a relatively lower levels of chlorophyll-a and chlorophyll-b. As the light-harvesting pigments in plant

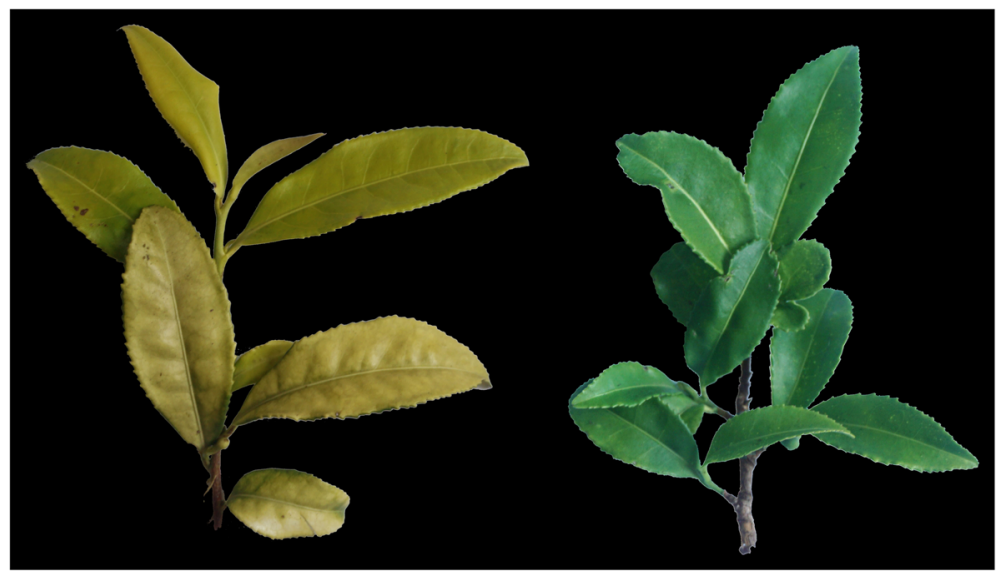

Fig. 1 Phenotypic characterization of chlorotic and green leaves under full sunlight (left) and shaded (right, 60\% light intensity) conditions 
Table 1 Concentrations of the main catechins and carotenoids in young shoots of chlorotic (full sunlight) and green (shaded) tea plants

\begin{tabular}{|c|c|c|}
\hline Name & $\begin{array}{l}\text { Shaded green } \\
\text { (G) plants }\end{array}$ & $\begin{array}{l}\text { Chlorotic } \\
\text { (C) plants }\end{array}$ \\
\hline \multicolumn{3}{|l|}{$\begin{array}{l}\text { Catechins (mg/g } \\
\text { fresh weight) }\end{array}$} \\
\hline Catechin gallate & $0.01 \pm 0.00 a$ & $0.06 \pm 0.01 b$ \\
\hline Gallate acid & $0.09 \pm 0.03$ & $0.16 \pm 0.03$ \\
\hline Gallocatechin & $0.26 \pm 0.04 a$ & $0.08 \pm 0.01 b$ \\
\hline Epicatechin & $1.73 \pm 0.12 a$ & $2.36 \pm 0.21 b$ \\
\hline Catechin & $0.08 \pm 0.02$ & $0.10 \pm 0.01$ \\
\hline Epigallocatechin & $0.59 \pm 0.04 a$ & $0.72 \pm 0.04 b$ \\
\hline Gallocatechin gallate & $0.29 \pm 0.05$ & $0.24 \pm 0.06$ \\
\hline Epigallocatechin gallate & $9.32 \pm 0.51 a$ & $7.82 \pm 1.1 b$ \\
\hline Epicatechin gallate & $5.24 \pm 0.23$ & $5.34 \pm 0.21$ \\
\hline \multicolumn{3}{|l|}{$\begin{array}{l}\text { Carotenoids and chlorophyll } \\
\left(\mathrm{\mu g} / \mathrm{cm}^{2} \text { leaf area) }\right.\end{array}$} \\
\hline Neoxanthin & $16.44 \pm 0.17 a$ & $1.21 \pm 0.03 b$ \\
\hline Lutein & $50.90 \pm 3.39 a$ & $11.15 \pm 0.12 b$ \\
\hline Violaxanthin & $3.45 \pm 0.11 a$ & $1.78 \pm 0.06 b$ \\
\hline$\beta$-Carotene & $8.08 \pm 0.14 a$ & $13.02 \pm 0.81 b$ \\
\hline Zeaxanthin & $3.22 \pm 0.17 a$ & $10.12 \pm 0.19 b$ \\
\hline Chlorophyll-a & $128.84 \pm 1.31 a$ & $5.83 \pm 0.16 b$ \\
\hline Chlorophyll-b & $34.46 \pm 2.26 a$ & $9.01 \pm 0.12 b$ \\
\hline
\end{tabular}

Different letters following data of the same lines indicate significant difference at $p<0.05$

photosynthesis system, carotenoids ensure efficient photosynthesis and prevent light damage for plants. In our study, the contents of lutein and neoxanthin reduced and with an increment of zeaxanthin and carotene in the chlorotic leaves (Table 1).

\section{Overview of metabolomic profiling}

A total of 1471 compounds were extracted from the raw data of the UPLC-TOF/MS analysis. We performed PLS-DA modeling to determine which metabolites were significantly affected by chlorosis (Fig. 2a). The components of the PLS-DA model explained $89.3 \%$ variance and the cumulative Q2 variance was $82.2 \%$ for the prediction accuracy, indicating the inter-group difference is significant. PLS-DA models were further confirmed by a 7-fold cross validation, and a permutation test was applied to validate the models' reliability rigorously (Fig. 2b, $n=200$ ). The score plots showed a clear separation between the chlorotic and the shaded green leaves with the first component (shown as the $\mathrm{X}$ axis in Fig. 2a). Differential metabolites were identified based on the VIP $>1.0$ and $\mid \mathrm{p}$ (corr) $\mid>0.5$ in the PLS-DA model and $p$ $<0.05$ in the $t$-test. Fifty-two differential metabolites were identified, which were responsible for flavonoid biosynthesis and phenylalanine metabolism (Table 2). These metabolites include flavan-3-ol (catechins), flavonols, flavonol glycosides, anthocyanin, and benzoic acid and derivatives.

\section{Differences in flavonoid metabolism between chlorotic and normal green leaves}

The chlorotic mutant showed lower expression levels of genes such as Phenylalanine ammonia-lyase (PAL), 4-coumarate-CoA ligase (4Cl), anthocyanidin reductase (ANR), chalcone synthase (CHS), flavanone 3-hydroxylase (F3H), flavonoid 3', 5'-hydroxylase (F3'5'H) and flavonol synthase (FLS) than did the shaded green leaves in the pathway from phenylalanine to epigallocatechin. However, chalcone isomerase (CHI) and flavonoid 3'-hydroxylase ( $F 3^{\prime} H$, the key gene of dihydroxy flavonoid synthetic) were up-regulated (Fig. 3) in the chlorotic leaves.

Analysis of metabolomics using UPLC-Q-TOF/MS revealed that the concentrations of flavan-3-ol, flavonols, and flavonol glycosides were greatly affected by shading (Table 2). Fifteen metabolites annotated as part of flavonoid metabolism were decreased in the chlorotic leaves compared with the green leaves, and these metabolites included (-)-epicatechin 8-C-glucoside, 6-galloylglucose, and epicatechin-ent-epicatechin. However, other flavonoids were not significantly reduced (e.g., epicatechin gallate, catechin) or were even increased (e.g., anthocyanidins, rutin, and catechin gallate) in the chlorotic leaves. It is interesting to note that the chlorotic leaves contained a higher level of dihydroxyflavonoids, mainly as quercetin and its glycosides (quercetin 3-galactoside,

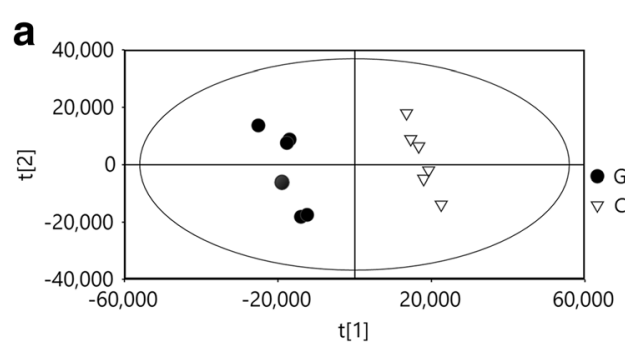

b

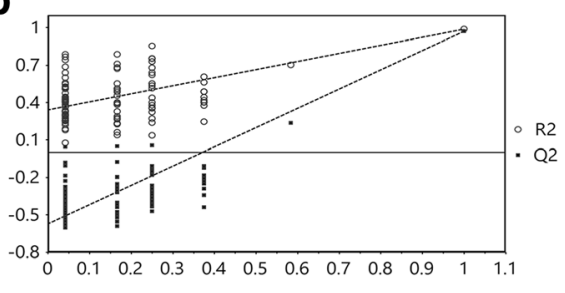

Fig. 2 PLS-DA score plots (a) and permutation test (b) of metabolites analyzed by UPLC-Q-TOF/MS in young tea shoots 
Table 2 Significantly changed (VIP $>1$ and $|p(c o r r)|>0.65$ from partial least squares discriminant analysis) intercellular metabolites induced by chlorosis

\begin{tabular}{|c|c|c|c|}
\hline Compounds & $V I P^{a}$ & $\mathrm{p}(\text { corr })^{b}$ & $\log 2(C / G)$ \\
\hline (-)-Epicatechin 8-C-glucoside & 1.57 & -0.97 & -1.86 \\
\hline 2,6-Digalloylglucose & 1.58 & -0.97 & -1.08 \\
\hline 3-(4-Hydroxybenzoyl) epicatechin & 1.52 & -0.94 & -1.27 \\
\hline 6-Galloylglucose & 1.48 & -0.88 & -2.10 \\
\hline Anthocyanidins & 1.54 & 0.95 & 2.10 \\
\hline Catechin 7-O-apiofuranoside & 1.35 & 0.84 & 0.75 \\
\hline Catechin-galacto & 1.38 & -0.86 & -0.48 \\
\hline Cyanidin 3-xyloside & 1.25 & 0.78 & 1.30 \\
\hline Delphinidin 3-glucoside & 1.16 & -0.72 & -1.64 \\
\hline Dihydromyricetin & 1.51 & 0.93 & 2.53 \\
\hline Gallocatechin 3-gallate & 1.60 & -0.99 & -1.17 \\
\hline Epicatechin 3-O-(3-O-methylgallate) & 1.54 & -0.96 & -1.69 \\
\hline Epicatechin-ent-epicatechin & 1.24 & -0.77 & -2.49 \\
\hline Catechingallate & 1.10 & 0.69 & 2.37 \\
\hline Epigallocatechin & 1.38 & 0.87 & 0.68 \\
\hline Kaempferol 3-(2G-apiosylrobinobioside) & 1.43 & -0.88 & -1.58 \\
\hline Kaempferol 7-(6"-galloylglucoside) & 1.59 & 0.99 & 1.38 \\
\hline Kaempferol-3-O-glucoside & 1.48 & 0.93 & 1.43 \\
\hline Kaempferol-3-O-rutinoside & 1.52 & -0.95 & -0.59 \\
\hline Leucodelphinidin & 1.45 & 0.89 & 1.54 \\
\hline Myricetin & 1.27 & -0.78 & -1.22 \\
\hline Myricetin 3-(3"-galloylrhamnoside) & 1.35 & -0.82 & -0.37 \\
\hline Myricetin 3,3'-digalactoside & 1.58 & -0.97 & -1.03 \\
\hline Myricetin 3-arabinoside & 1.29 & -0.80 & -1.73 \\
\hline Naringenin-4'-O-glucuronide & 1.55 & 0.95 & 1.33 \\
\hline Naringin & 1.54 & 0.93 & 1.49 \\
\hline Procyanidin C1 & 1.41 & -0.87 & -1.21 \\
\hline Quercetagetin 3'-methylether 7-glucoside & 1.55 & 0.94 & 1.81 \\
\hline Quercetin & 1.63 & 0.99 & 1.32 \\
\hline Quercetin 3-(3R-glucosylrutinoside) & 1.57 & 0.97 & 1.59 \\
\hline Quercetin 3,7,4'-O-triglucoside & 1.60 & 0.99 & 1.24 \\
\hline Quercetin 3-arabinoside & 1.54 & 0.95 & 2.42 \\
\hline Quercetin 3-galactoside & 1.63 & 0.99 & 0.48 \\
\hline Quercetin 3-O-alpha-D-arabinopyranoside & 1.61 & 0.99 & 1.63 \\
\hline Quercetin 3-rutinoside 7-galactoside & 1.53 & 0.95 & 4.35 \\
\hline Quercetin 4',7-diglucoside & 1.64 & 1.00 & 1.25 \\
\hline Quercetin-3'-glucuronide & 1.63 & 0.99 & 1.21 \\
\hline Rutin & 1.60 & 0.99 & 1.35 \\
\hline Cinnamic acid & 1.63 & 0.99 & 0.85 \\
\hline Chicoric acid & 1.53 & -0.92 & -2.17 \\
\hline Fertaric acid & 1.53 & -0.94 & -1.25 \\
\hline p-Coumaric acid & 1.29 & -0.81 & -0.73 \\
\hline
\end{tabular}

Table 2 Significantly changed (VIP $>1$ and $|p(c o r r)|>0.65$ from partial least squares discriminant analysis) intercellular metabolites induced by chlorosis (Continued)

\begin{tabular}{lrrr}
\hline Benzoic acid & 1.77 & 0.92 & 0.81 \\
Gallic acid & 1.50 & 0.91 & 1.02 \\
4-Hydroxybenzoic acid & 1.38 & 0.85 & 1.72 \\
Glucogallina & 1.55 & -0.79 & -0.25 \\
Chalcones & 1.46 & 0.94 & 1.24 \\
Caffeic acid & 1.37 & -0.90 & -2.13 \\
Frerulic acid & 1.24 & 0.87 & 0.84 \\
Quinine & 1.77 & 0.92 & 3.04 \\
Quinic acid & 1.34 & -0.83 & -1.73 \\
Shikimic acid & 1.16 & -0.73 & -0.32 \\
\hline
\end{tabular}

${ }^{a}$ VIP means variable importance in the projection values from partial least squares discriminant analysis (PLS-DA)

${ }^{b} \mathrm{p}$ (corr) indicates loadings scaled as a correlation coefficient (ranging from -1.0 to 1.0$)$

${ }^{c} \mathrm{C} / \mathrm{G}$ means the ratio of the mean peak intensity in chlorotic ( $\mathrm{C}$, full sun light) and green $(G$, shaded) tea plants

quercetin 4',7-diglucoside, quercetin 3-rutinoside 7galactoside etc.) than the green leaves, whereas the chlorotic leaves possessed a lower level of myricetrin glycoside (myricetin 3-arabinoside, myricetin 3,3'-digalactoside etc.) than did the green leaves (Table 2).

In the validation of quantitative analysis using HPLC, the chlorotic leaves showed lower contents of trihydroxy catechins (particularly the gallocatechin, epigallocatechin gallate) compared to shaded green leaves. However, the contents of dihydroxy catechins (catechin gallate, epicatechin) were significantly higher in the chlorotic leaves than in the shaded green shoots (Table 1).

Furthermore, metabolites involved in the metabolism of benzenoids, phenylpropanoids and polyketides, the branch upstream of catechin and flavonoid metabolism, were significantly downregulated in the chlorotic leaves (Table 2). Such as coumaric acid, caffeic acid, quinic acid, shikimic acid and chicoric acid.

\section{The intracellular localization of flavonoids and Gene expression related to the antioxidant system in chlorotic tea leaves}

The intracellular localization of flavonoids (mainly dihydroxy flavonoids) was highly different between the chlorotic and shaded green leaves (Fig. 4). The flavonoids (Fig. 4, green fluorescent signal) were mainly located in the epidermal leaf cells and in the light-receiving area of leaves, whereas chlorophyll (green and red signal in Fig. 4) and catechins (Fig. 4, red signal) were distributed throughout the cells of the shaded green leaves. However, the catechin signal (Fig. 4, red signal) was significantly weakened, the chloroplasts degraded (Fig. 4, arrows), and the dihydroxy flavonoids spread to all leaf cells as the leaves became chlorotic. 


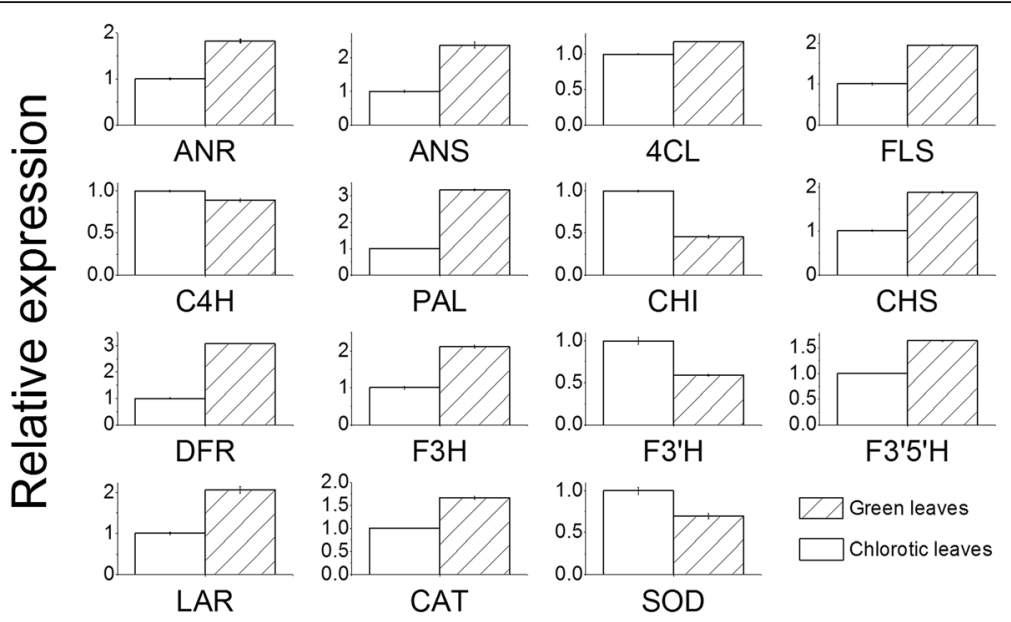

Fig. 3 Quantitative RT-PCR validation. PAL, phenylalanine ammonia-lyase; $\mathrm{C} 4 \mathrm{H}$, cinnamate 4-hydroxylase; 4Cl, 4-coumarate-CoA ligase; $\mathrm{CHI}$, chalcone isomerase; CHS, chalcone synthase; F3'5'H, flavonoid 3', 5'-hydroxylase; F3H, flavanone 3-hydroxylase; F3'H, flavonoid 3'-monooxygenase; FLS, flavonol synthase; DFR, dihydroflavonol-4-reductase; ANR, anthocyanidin reductase; ANS, anthocyanidin synthase; LAR, leucoanthocyanidin reductase; CAT: catalase; SOD, superoxide dismutase

The expression level of the gene encoding catalase (CAT) was 1.4-fold higher in shaded green leaves than in chlorotic leaves (Fig. 3). By contrast, the gene encoding superoxide dismutase (SOD) were repressed in chlorotic leaves.

\section{Discussion}

A schematic flow chat of the phenylpropanoid/flavonoid pathway affected by the chlorosis mutation is shown in Fig. 5. The lower contents were accompanied by the reduced expression levels of most genes (except $\mathrm{CHI}$ and $F 3^{\prime} H$ ) involved in the pathway starting from phenylalanine to these metabolites. Furthermore, the expression levels of genes involved in the upstream shikimic acid pathway, which leads to the biosynthesis of phenylalanine, were also strongly down-regulated (Fig. 5). Such findings suggest that flavonoid biosynthesis was strongly inhibited in the chlorotic mutant. One of the explanations for such inhibition lies in the destruction of the chloroplasts, which are the sites of flavonoid biosynthesis [32]. The present work showed that the location of flavonoids in the tissue changed as the leaves became chlorotic, as the anabolic metabolism of flavonoids is coupled with the chloroplast (Fig. 4). Furthermore, the reduced accumulation of flavonoids could be attributed to the low glucose content of the chlorotic mutant. This

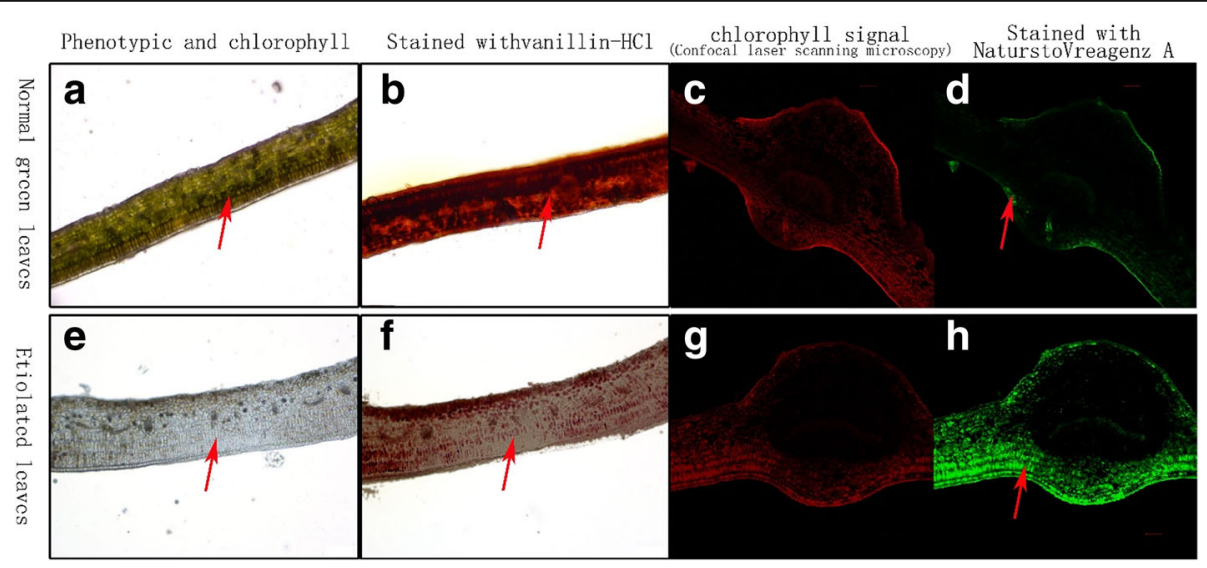

Fig. 4 Histochemical localization of catechin and flavonol in Huangjinya leaves. a, b, c, d show the normal green leaves of Huangjinya shaded with $60 \%$ (NG); $\mathbf{e}, \mathbf{f}, \mathbf{g}, \mathbf{h}$ show the chlorotic leaves of HJY exposed to $100 \%$ sunlight (EM); $\mathbf{b}$ and $\mathbf{f}$ show staining with vanillin- $\mathrm{HCl}$ reagent; the red signal represents phenolic compounds (mainly catechins; $\mathbf{d}$ and $\mathbf{h}$ were stained with NaturstoVreagenz A (confocal laser scanning microscopy), and the green fluorescence signal represents flavonol 


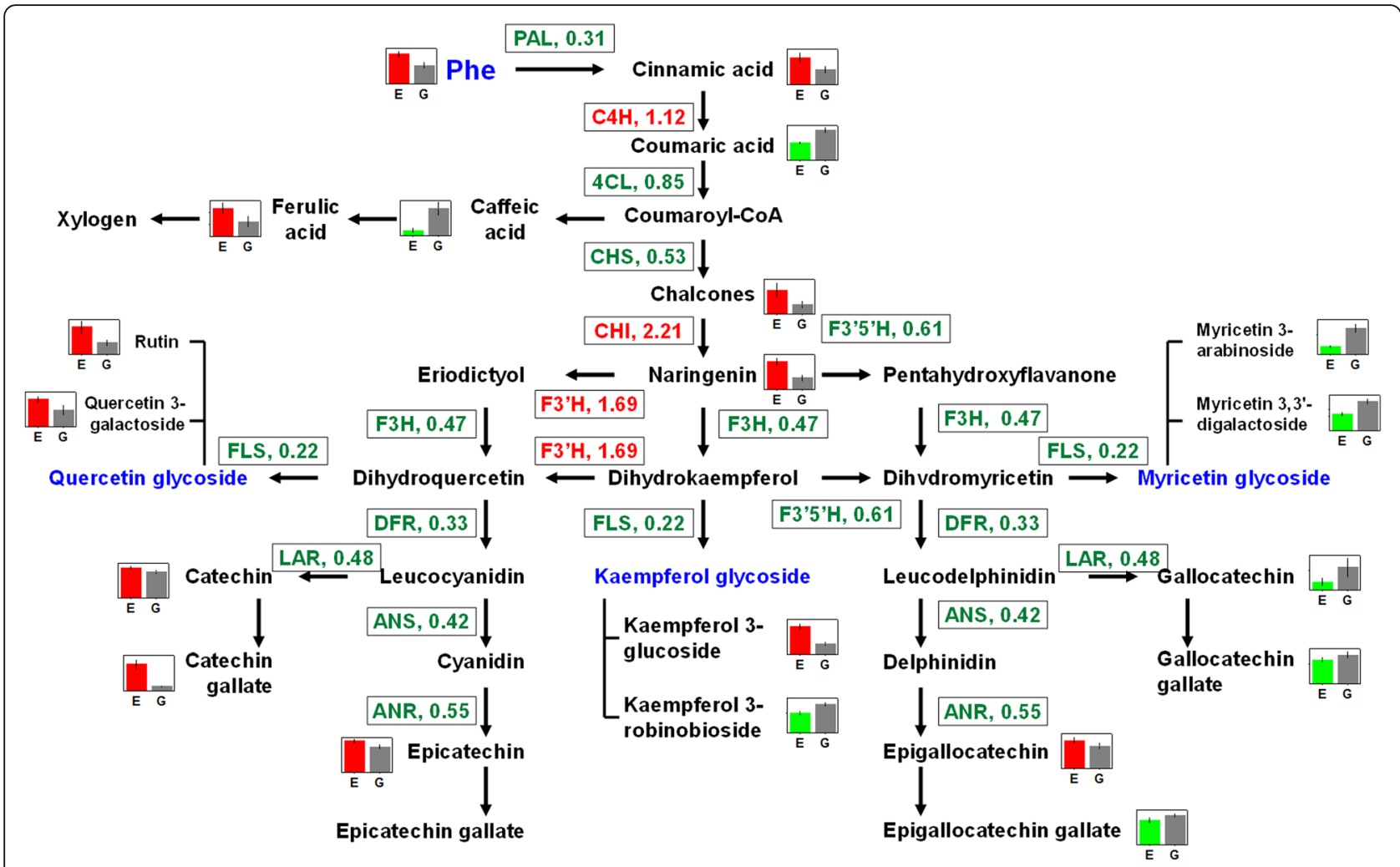

Fig. 5 Schematic representation of phenylpropanoid/flavonoid pathway as affected by the chlorosis mutation. Red and green font indicate up- and downregulated genes, respectively, in the chlorotic leaves compared to shaded plants. Significantly changed metabolites are shown with a barplot (E and G indicate chlorotic and green leaves, respectively) while compounds that were not identified in this study are shown in gray font. PAL, phenylalanine ammonia-lyase; C4H, cinnamate 4-hydroxylase; 4Cl, 4-coumarate-CoA ligase; CHI, chalcone isomerase; CHS, chalcone synthase; F3'5'H, flavonoid 3', 5'-hydroxylase; F3H, flavanone 3-hydroxylase; F3'H, flavonoid 3'-monooxygenase; FLS, flavonol synthase; DFR, dihydroflavonol-4-reductase; ANR, anthocyanidin reductase; ANS, anthocyanidin synthase; LAR, leucoanthocyanidin reductase

finding corroborates the findings of Yang et al. [39], who showed that the metabolism of shikimic acid, prephenic acid, and phenylpyruvic acid was strongly inhibited in dark-induced chlorotic tea plants. The decrease of sugars also negatively affected the glycosylation of catechins, as well as the synthesis of flavonoid glycosides [4].

The accumulation of small molecules with antioxidative activity plays important roles in mitigating ROS accumulation [40]. These low-molecular-weight antioxidants include sugars, a-tocopherols, glutathione, amino acids (e.g., proline), ascorbic acid, carotenoids and quinic acid derivatives (e.g., chlorogenic acid). In our study, the tea mutant did not develop functional chloroplasts (data not shown) and lacked chlorophyll under light conditions (Table 1), suggesting that the mutant without shading was exposed to light stress. Many phenylpropanoid [27] and anthocyanin [28] compounds are effective sunlight attenuators and protect photosynthetic organs (chloroplasts) faced with a superabundance of radiant energy. Flavonoids play a vital role in protecting plants to excess UV-B or sunlight irradiance [29-31]. Flavonoids such as quercetin and dihydroxy catechins also serve multiple functions in higher plants under distinct environmental conditions [26]. Flavonoids with a dihydroxy structure in the B-ring accumulate preferentially in response to high doses of ultraviolet radiation (UV) or sunlight radiation $[10,32$, 33]. Therefore, the physiological mechanisms in leaves enduring light stress may be associated with dihydroxy flavonoids. In our study, chlorotic leaves contained higher levels of dihydroxyflavonoids, mainly as catechins (catechin gallate, epicatechin) and quercetin and its glycosides, whereas the levels of myricetrin and kaempferol glycoside were lower than in green leaves (Fig. 5, Tables 1 and 2). The similar results have been observed under sun light and partially shading treatments for normal tea plants [5], which suggest that the functions of flavonoids to ROS are not only available in chlorotic tea leaves, but also in all normal tea species. We also found that the dihydroxy flavonoids were mainly distributed intracellularly in the leaf epidermal cells and in the light-receiving area, whereas chlorophyll and catechin were distributed throughout the cells of the chlorotic tea mutant. 
Generally, plants possess diverse photoprotection mechanisms, including the dissipation of absorbed light energy as thermal energy by non-photochemical quenching (NPQ). Photoinhibitory quenching (qI), quenching due to state transitions (qT), and high-energy-statequenching (qE) are the three main components of NPQ, among which $\mathrm{qI}$ is related to the slow conversion of the xanthophyll cycle pigment zeaxanthin to violaxanthin, while $\mathrm{qE}$ depends on the existence of special xanthophyll molecules (i.e., lutein and zeaxanthin) bound to the PSII antenna proteins $[41,42]$. We observed a significant increase in the zeaxanthin and carotene contents in the chlorotic leaves, although the total content of carotenoids decreased by $54 \%$ (Table 1). The xanthophyll cycle protects plants from high light intensity by converting violaxanthin into zeaxanthin, which participates in the thermal dissipation of excess absorbed light energy [34]. We also noted higher transcript levels of violaxanthin de-epoxidase and zeaxanthin epoxidase (EC: 1.10.99.3, 1.14.13.90, a key gene in xanthophyll cycle that protects plants from high-intensity light) in chlorotic leaves compared with green (shaded) leaves with transcriptomic analysis (data not shown here). Suggesting that the xanthophyll cycle plays an important role in protecting 'Huangjinya' from high light intensity. This result is highly consistent with the findings of Li et al. [20]. The tea mutants 'White leaf No.1' and 'Huangjinya' both showed reduced carotenoid and zeaxanthin content in chlorotic leaves compared with 'Fuding dabaicha' $[17,43]$. Thus, 'Huangjinya' shows a significant difference in carotenoid levels compared with other tea mutants (mainly the albino mutants). We hypothesized that the variation in carotenoid composition and biosynthesis is a specific light protective mechanism in light-sensitive tea mutants. However, serious damage to cell membrane structures in chlorotic leaves suggests that antioxidants and the xanthophyll cycle were insufficient to protect tea plants from photodamage.

Under abiotic stresses, including light stress, highly reactive and toxic ROS such as superoxide anion radicals, singlet oxygen, hydrogen peroxide $\left(\mathrm{H}_{2} \mathrm{O}_{2}\right)$, and hydroxyl radicals $\left({ }^{\circ} \mathrm{OH}\right)$ are induced (Fig. 6), and they are mitigated by ROS scavenging systems [24]. Chloroplasts respond to ROS via a significant change in composition, resulting in rapid morphological and functional modifications [44]. The inactivation of the ROS scavenging system and the biofilm protection function in the chloroplast may cause damage to the membrane structure and the disintegration of chloroplasts. Chloroplasts react violently to ROS generated by high-intensity light [23]. CAT is an iron porphyrin prosthetic groupconjugated enzyme that scavenges ROS [45]. In our study, gene encoding CAT activity down-regulated and iron content decreased $(1.6 \mathrm{mg} / \mathrm{kg}$ in chlorotic and $1.1 \mathrm{mg} / \mathrm{kg}$ in green leaves) in chlorotic leaves. These results suggest that CAT synthesis might be restricted by iron utilization and chelation.

\section{Conclusions}

In this study, the variation in gene expression patterns and metabolites between chlorotic leaves and normal green leaves (leaves under shading treatment) in the 'Huangjinya' tea mutant was uncovered using metabolomics analysis. Our results suggest that high-intensity light stress caused photooxidation in chlorotic leaves and induced multi-operational photoprotection mechanisms for scavenging ROS, including the activation of dihydroxy flavonoids and xanthophyll cycle pathways, which also reversed the photodamage in mutant leaves, helping them endure light stress. Moreover, the differential accumulation of metabolites and differential gene expression suggested that dihydroxy flavonoids have great differences in terms of their functional roles in tea

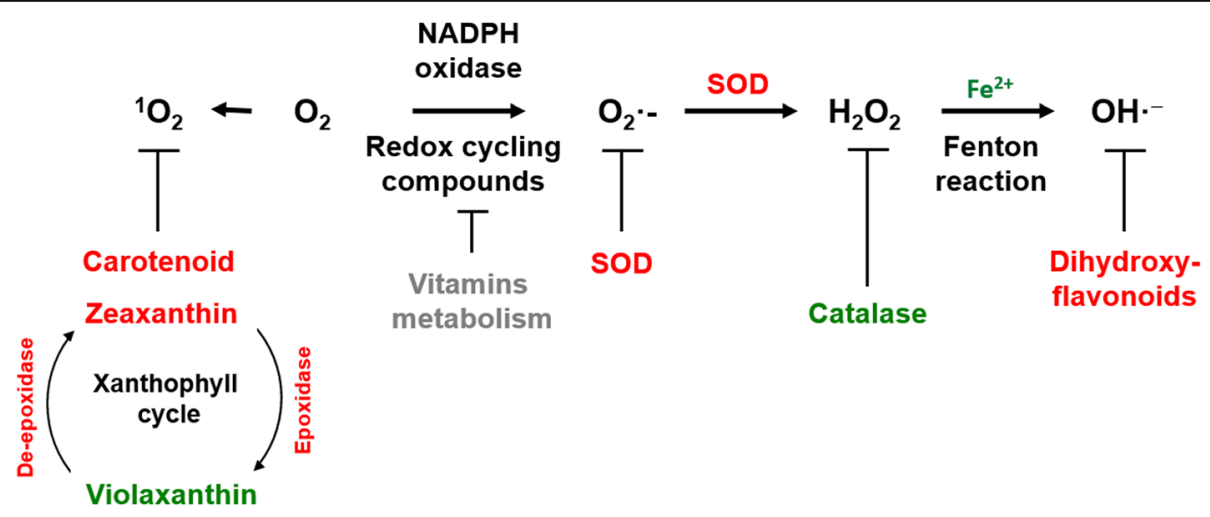

Fig. 6 Production and scavenging of free radicals. Red and green font indicate up- and down-regulated genes / metabolites in the chlorotic leaves compared to shaded plants 
leaves. Especially, the accumulation and location of quercetin and its glycosides (compare with myricetrin and kaempferol glycoside) in chlorotic leaves suggest their great contribution to photo-protection and their unique functional roles in ROS scavenging in tea leaves.

\section{Additional file}

Additional file 1: Table S1. Primer sequences for quantitative RT-PCR of genes related to the antioxidant system in chlorotic tea leaves. (DOCX $16 \mathrm{~kb}$ )

\begin{abstract}
Abbreviations
OH: hydroxyl radicals; 4Cl: 4-coumarate-CoA ligase; ANR: anthocyanidin reductase; ANS: anthocyanidin synthase; C4H: cinnamate 4-hydroxylase; CAT: Catalase; CHI: Chalcone isomerase; CHS: Chalcone synthase; DFR: Dihydroflavonol-4reductase; F3'5'H: flavonoid 3', 5'-hydroxylase; F3H: Flavanone 3-hydroxylase; F3' $\mathrm{H}$ : Flavonoid 3'-monooxygenase; FLS: Flavonol synthase; $\mathrm{H}_{2} \mathrm{O}_{2}$ : Hydrogen peroxide; HPLC: High-performance liquid chromatography; LAR: Leucoanthocyanidin reductase; NPQ: Non-photochemical quenching; PAL: Phenylalanine ammonialyase; PLS-DA: Projection to latent structure discriminant analysis; PSII: Photosystem Il; qE: High-energy-state-quenching; ql: Photoinhibitory quenching; qRTPCR: Quantitative real-time PCR; $\mathrm{qT}$ : Quenching due to state transitions; ROS: Reactive oxygen species; SOD: Superoxide dismutase; UPLC-Q-TOF/ MS: Ultra-performance liquid chromatography quadrupole time-of-flight mass spectrometry; UV: Ultraviolet radiation; VIP: Variable importance in the projection
\end{abstract}

\section{Acknowledgements}

Thanks are given to Prof. Zongmao Chen and Dr. Xinzhong Zhang for assistance in using the UPLC-Q-TOF/MS and Prof. Kairong Wang for providing the mutant clone.

\section{Funding}

This work was financially supported by the Chinese Academy of Agricultural Sciences through Agricultural Sciences Innovation Project (CAAS-ASTIP-2016TRICAAS) and the Ministry of Agriculture of China through the Earmarked Fund for the China Agriculture Research System (CARS 23).

\section{Availability of data and materials}

All data generated or analysed during this study are included in this published article and its supplementary information files.

\section{Authors' contributions}

QFZ and MYL gathered samples, participated in the study design, performed sample analysis, interpreted the results and drafted the manuscript. JYR conceived of the study, provided funding, gave guidance on experimental design and revised the manuscript. All authors read and approved the final manuscript.

\section{Competing interests}

The authors declare that they have no competing interests.

\section{Consent for publication}

Not applicable.

\section{Ethics approval and consent to participate} Not applicable.

\section{Publisher's Note}

Springer Nature remains neutral with regard to jurisdictional claims in published maps and institutional affiliations.
Received: 14 November 2016 Accepted: 9 March 2017

Published online: 20 March 2017

\section{References}

1. Zhang Q, Ruan J. Tea: analysis and tasting. In: Caballero B, Finglas PM, Toldrá F, editors. Encyclopedia of food and health. Oxford: Academic; 2015. p. 256-67.

2. Cheynier V, Comte G, Davies KM, Lattanzio V, Martens S. Plant phenolics: recent advances on their biosynthesis, genetics, and ecophysiology. Plant Physiol Biochem. 2013;72:1-20.

3. Fiqueiredo-Gonzalez M, Cancho-Grande B, Boso S, Santiago JL, Martinez MC, Simal-Gandara J. Evolution of flavonoids in Mouraton berries taken from both bunch halves. Food Chem. 2013;138:1868-77.

4. Wang Y, Gao L, Wang Z, Liu Y, Sun M, Yang D, Wei C, Shan Y, Xia T. Lightinduced expression of genes involved in phenylpropanoid biosynthetic pathways in callus of tea (Camellia sinensis (L.) O. Kuntze). Sci Hortic. 2012;133:72-83

5. Zhang Q, Shi Y, Ma L, Yi X, Ruan J. Metabolomic analysis using UltraPerformance Liquid Chromatography-Quadrupole-Time of Flight Mass Spectrometry (UPLC-Q-TOF/MS) uncovers the effects of light intensity and temperature under shading treatments on the metabolites in tea. PLoS One. 2014;9:e112572.

6. Tattini M, Guidi L, Morassi-Bonzi L, Pinelli P, Remorini D, Degl'Innocenti E, Giordano C, Massai R, Agati G. On the role of flavonoids in the integrated mechanisms of response of Ligustrum vulgare and Phillyrea latifolia to high solar radiation. New Phytologist. 2005:167:457-70.

7. Agati G, Biricolti S, Guidi L, Ferrini F, Fini A, Tattini M. The biosynthesis of flavonoids is enhanced similarly by UV radiation and root zone salinity in $\mathrm{L}$. vulgare leaves. J Plant Physiol. 2011;168(3):204-12.

8. Jay-Allemand C, Tattini M, Gould K. New evidence for the functional roles of secondary metabolites in plant-environment interactions. Environ Exp Bot. 2015;119:1-3

9. Landi M, Tattini M, Gould K. Multiple functional roles of anthocyanins in plant-environment interactions. Environ Exp Bot. 2015;119:4-17.

10. Tattini M, Galardi C, Pinelli P, Massai R, Remorini D, Agati G. Differential accumulation of flavonoids and hydroxycinnamates in leaves of Ligustrum vulgare under excess light and drought stress. New Phytologist. 2004;63:547-61.

11. Agati G, Azzarello E, Pollastri S, Tattini M. Flavonoids as antioxidants in plants: location and functional significance. Plant Sci. 2012;196:67-76.

12. Satou M, Enoki H, Oikawa A, Ohta D, Saito K, Hachiya T, Sakakibara H, Kusano M, Fukushima A, Saito K, Kobayashi M, Nagata N, Myouga F, Shinozaki K, Motohashi R. Integrated analysis of transcriptome and metabolome of Arabidopsis albino or pale green mutants with disrupted nuclear-encoded chloroplast proteins. Plant Mol Biol. 2014;85:411-28.

13. Smith GJ, Markham KR. Tautomerism of flavonol glucosides - relevance to plant UV protection and flower colour. J Photochem Photobiol A Chem. 1998:118:99-105.

14. Yamasaki H, Sakihama Y, Ikehara N. Flavonoid-peroxidase reaction as a detoxification mechanism of plant cell against $\mathrm{H} 2 \mathrm{O} 2$. Plant Physiol. 1997;115:1405-17.

15. Neill SO, Gould KS. Anthocyanins in leaves: light attenuators or antioxidants? Funct Plant Biol. 2003;30:865-73.

16. Ma C-L, Chen L, Wang X-C, Jin J-Q, Ma J-Q, Yao M-Z, Wang Z-L. Differential expression analysis of different albescent stages of 'Anji Baicha' (Camellia sinensis (L.) O. Kuntze) using cDNA microarray. Sci Hortic. 2012;148:246-54.

17. Feng L, Gao MJ, Hou RY, Hu XY, Zhang L, Wan XC, Wei S. Determination of quality constituents in the young leaves of albino tea cultivars. Food Chem. 2014;155:98-104.

18. Li Q, Huang J, Liu S, Li J, Yang X, Liu Y, Liu Z. Proteomic analysis of young leaves at three developmental stages in an albino tea cultivar. Proteome Sci. 2011;9:44

19. Xiong L, Li J, Li Y, Yuan L, Liu S, Huang J, Liu Z. Dynamic changes in catechin levels and catechin biosynthesis-related gene expression in albino tea plants (Camellia sinensis L.). Plant Physiol Biochem. 2013;71:132-43.

20. Li N, Yang Y, Ye J, Lu J, Zheng X, Liang Y. Effects of sunlight on gene expression and chemical composition of light-sensitive albino tea plant. Plant Growth Regul. 2015;78:253-62.

21. Chen M, Chory J, Fankhauser C. Light signal transduction in higher plants. Annu Rev Genet. 2004;38:87-117. 
22. Lee LS, Choi JH, Son N, Kim SH, Park JD, Jang DJ, Jeong Y, Kim HJ. Metabolomic analysis of the effect of shade treatment on the nutritional and sensory qualities of green tea. J Agric Food Chem. 2013;61:332-8.

23. Alboresi A, Dall'Osto L, Aprile A, Carillo P, Roncaglia E, Cattivelli L, Bassi R. Reactive oxygen species and transcript analysis upon excess light treatment in wild-type Arabidopsis thaliana vs a photosensitive mutant lacking zeaxanthin and lutein. BMC Plant Biol. 2011;11:62.

24. Nishiyama Y, Murata N. Revised scheme for the mechanism of photoinhibition and its application to enhance the abiotic stress tolerance of the photosynthetic machinery. Appl Microbiol Biotechnol. 2014;98:8777-96.

25. Aluru M, Zola J, Foudree A, Rodermel S. Chloroplast photooxidation-induced transcriptome reprogramming in Arabidopsis immutans white leaf sectors. Plant Physiol. 2009;150:904-23.

26. Agati G, Matteini P, Goti A, Tattini M. Chloroplast-located flavonoids can scavenge singlet oxygen. New Phytologist. 2007;174:77-89.

27. Agati G, Tattini M. Multiple functional roles of flavonoids in photoprotection. New Phytologist. 2010;186:786-93.

28. Gould KS, Dudle DA, Neufeld HS. Why some stems are red: cauline anthocyanins shield photosystem II against high light stress. J Exp Bot. 2010;61:2707-17.

29. Jordan BR. Review: molecular response of plant cells to UV-B stress. Funct Plant Biol. 2002;29:909-16.

30. Neill SO, Gould KS, Kilmartin PA, Mitchell KA, Markham KR. Antioxidant capacities of green and cyanic leaves in the sun species, Quintinia serrata. Functional Plant Biology. 2002;29:1437-43.

31. Bernal M, Llorens L, Julkunen-Tiitto R, Badosa J, Verdaguer D. Altitudinal and seasonal changes of phenolic compounds in Buxus sempervirens leaves and cuticles. Plant Physiol Biochem. 2013;70:471-82.

32. Agati G, Galardi C, Gravano E, Romani A, Tattini M. Flavonoid distribution in tissues of Phillyrea latifolia L. leaves as estimated by microspectrofluorometry and multispectral fluorescence microimaging. Photochem Photobiol. 2002;76:350-60.

33. Hofmann R, Campbell B, Bloor S, Swinny E, Markham K, Ryan K, Fountain D. Responses to UV-B radiation in Trifolium repens L.-physiological links to plant productivity and water availability. Plant. Cell \& Environment. 2003;26:603-12.

34. Buchanan BB, Gruissem W, Jones RL. Biochemistry \& molecular biology of plants. Rockville: American Society of Plant Physiologists; 2000.

35. Roelfsema MRG, Konrad KR, Marten H, Psaras GK, Hartung W, Hedrich R. Guard cells in albino leaf patches do not respond to photosynthetically active radiation, but are sensitive to blue light, $\mathrm{CO} 2$ and abscisic acid. Plant Cell Environ. 2006;29:1595-605.

36. Wu C, Xu H, Héritier J, Andlauer W. Determination of catechins and flavonol glycosides in Chinese tea varieties. Food Chem. 2012;132:144-9.

37. Liu Y, Gao L, Xia T, Zhao L. Investigation of the site-specific accumulation of catechins in the tea plant (Camellia sinensis (L.) O. Kuntze) via vanillin- $\mathrm{HCl}$ staining. J Agric Food Chem. 2009;57:10371-6.

38. Hutzler P, Fischbach R, Heller W, Jungblut TP, Reuber S, Schmitz R, Veit M, Weissenbock G, Schnitzler JP. Tissue localization of phenolic compounds in plants by confocal laser scanning microscopy. J Exp Bot. 1998;49:953-65.

39. Yang Z, Kobayashi E, Katsuno T, Asanuma T, Fujimori T, Ishikawa T, Tomomura M, Mochizuki K, Watase T, Nakamura Y, Watanabe N. Characterisation of volatile and non-volatile metabolites in etiolated leaves of tea (Camellia sinensis) plants in the dark. Food Chem. 2012;135:2268-76.

40. Sharma P, Jha AB, Dubey RS, Pessarakli M. Reactive Oxygen Species, Oxidative Damage, and Antioxidative Defense Mechanism in Plants under Stressful Conditions. J Bot. 2012;48:909.

41. Takahashi S, Badger MR. Photoprotection in plants: a new light on photosystem II damage. Trends Plant Sci. 2011;16:53-60.

42. Kusama $Y$, Inoue $S$, Jimbo $H$, Takaichi $S$, Sonoike $K$, Hihara $Y$, Nishiyama $Y$. Zeaxanthin and echinenone protect the repair of photosystem II from inhibition by singlet oxygen in synechocystis sp. PCC 6803. Plant Cell Physio. 2015;156:906-16.

43. Wang L, Yue C, Cao H, Zhou Y, Zeng J, Yang Y, Wang X. Biochemical and transcriptome analyses of a novel chlorophyll-deficient chlorina tea plant cultivar. BMC Plant Biol. 2014;14:352

44. Xing F, Li Z, Sun A, Xing D. Reactive oxygen species promote chloroplast dysfunction and salicylic acid accumulation in fumonisin B1-induced cell death. Febs Letters. 2013;587:2164-72.

45. Vanderauwera S, Zimmermann $P$, Rombauts $S$, Vandenabeele $S$, Langebartels C, Gruissem W, Inze D, Van Breusegem F. Genome-wide analysis of hydrogen peroxide-regulated gene expression in Arabidopsis reveals a high light-induced transcriptional cluster involved in anthocyanin biosynthesis. Plant Physiol. 2005;139:806-21.

\section{Submit your next manuscript to BioMed Central and we will help you at every step:}

- We accept pre-submission inquiries

- Our selector tool helps you to find the most relevant journal

- We provide round the clock customer support

- Convenient online submission

- Thorough peer review

- Inclusion in PubMed and all major indexing services

- Maximum visibility for your research

Submit your manuscript at www.biomedcentral.com/submit 